\title{
Enhancing Medical Images by New Fuzzy Membership Function Median Based Noise Detection and Filtering Technique
}

\author{
G. Elaiyaraja ${ }^{\dagger}$ and N.Kumaratharan*
}

\begin{abstract}
In recent years, medical image diagnosis has growing significant momentous in the medicinal field. Brain and lung image of patient are distorted with salt and pepper noise is caused by moving the head and chest during scanning process of patients. Reconstruction of these images is a most significant field of diagnostic evaluation and is produced clearly through techniques such as linear or non-linear filtering. However, restored images are produced with smaller amount of noise reduction in the presence of huge magnitude of salt and pepper noises. To eliminate the high density of salt and pepper noises from the reproduction of images, a new efficient fuzzy based median filtering algorithm with a moderate elapsed time is proposed in this paper. Reproduction image results show enhanced performance for the proposed algorithm over other available noise reduction filtering techniques in terms of peak signal -to -noise ratio (PSNR), mean square error (MSE), root mean square error (RMSE), mean absolute error (MAE), image enhancement factor (IMF) and structural similarity (SSIM) value when tested on different medical images like magnetic resonance imaging (MRI) and computer tomography (CT) scan brain image and CT scan lung image. The introduced algorithm is switching filter that recognize the noise pixels and then corrects them by using median filter with fuzzy two-sided $\pi$-membership function for extracting the local information.
\end{abstract}

Keywords: CT scan brain image, Fuzzy switching median filter, Image enhancement factor, MRI scan brain image, Peak signal- to-noise ratio, Salt and pepper noise.

\section{Introduction}

Medical images are distorted by salt and pepper noise is largely caused by the acquisition or transmission of digital images in a noisy channel [13]. Salt and pepper noise is one type of impulse noise which can degrade the image visualization, where the noisy pixels can take only the minimum and maximum pixel elements of image. The linear filter like mean filter and interrelated filters are not efficient for removal of salt and pepper noise. Non-linear filtering techniques like standard median filter (SMF) and Adaptive median filter (AMF) are widely used to remove salt and pepper noise [21]. However, the SMF, which restores each pixel with median pixel value in the filtered image, exhibits blurring of reconstructed image [3]. SMF is effective only at low noise densities. Several techniques have been implemented for removal of salt and pepper noise at high noise densities [1-9].

Recent methods like efficient detail-preserving approach (EDPA) and efficient edge-preserving algorithm (EEPA) based on alpha-trimmed mean statistical estimator have

$\dagger$ Corresponding Author: Dept. of Electronics and Communication Engineering, Adhiparasakthi College of Engineering, G.B.Nagar, Tamilnadu, India. (elaiyarg@gmail.com)

* Dept. of Information and Technology, Srivenkateswara College of Engineering, Sriperumbudur, Tamilnadu, India. (kumaratharan@ rediffmail.com)

Received: August 6, 2014; Accepted: June 24, 2015 been proposed for the removal of salt-and-pepper noise without degrading image fine information's [2]. Then, adaptive impulse detector with center-weighted median (ACWM) filter efficiently to remove salt-and-pepper noise. These techniques simply perform well when an image is degraded with $50 \%$ of noise densities and small window size. On the other hand, Progressive Switching Median Filter(PSMF), decision based algorithm (DBA), modified decision based algorithm (MDBA), are one of the fastest and efficient algorithms of capable of salt-andpepper noise removal at high noise densities as $80 \%$ [18]. A most important disadvantage of this algorithm is striping problem at high noise densities. To overcome this drawback, modified decision based unsymmetric trimmed median filter (MDBUTMF) is used to remove salt-andpepper noise at very high densities as $80-90 \%$. In response to this noise problem, the switching median filter [6], in which an impulse detector of employed to classify the center pixel of the filtering window is proposed. In the filtering algorithm, at high noise density, the processing pixel is replaced by the mean value of pixel elements within the window [5]. This causes blurring of fine image details or high computational time [10]. Examining all those problems exist in literature, we have proposed a new switching based algorithm, which aids to accurate medical image visualization. This algorithm gives better metric values than the existing median based algorithms [2-6]. 


\section{Proposed Algorithm}

The modified fuzzy switching median based filter is a non-causal two-stage filter, one stage for finding the pixels are noise-free or noise pixels and second stage for noise reduction as shown in figure Fig. 1. The first step to detect the two salt-and-pepper noise intensities. When a "corrupted pixel" is discovered, it is switched to the next filtering stage [11]. Otherwise, when a pixel is marked as "uncorrupted pixel" it will be left unchanged and the filtering process is spared to avoid replacing any fine details and textures in the noisy image [4].

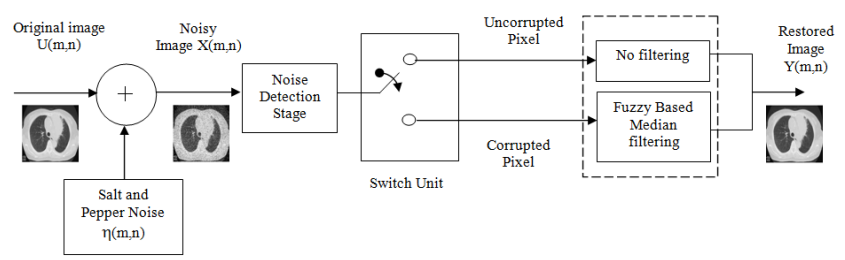

Fig. 1. Proposed medical image enhancing structure

\subsection{Switching noise detection process}

The filter initially estimates the two salt-and-pepper noise intensities of histogram of noise image shown in Fig. 2, and then the detection stage starts by searching for the two local maximums, Lsalt $=255$ and Lpepper $=0$, representing the two salt-and-pepper noise intensities starting from both ends of noisy image histogram [17]. These two salt-and-pepper noise pixels will be used to recognize possible "corrupted pixels" in the image as shown in Fig. 3. A binary noise values $B(m, n)$ will be formed based on noise pixels as shown Fig. 4.

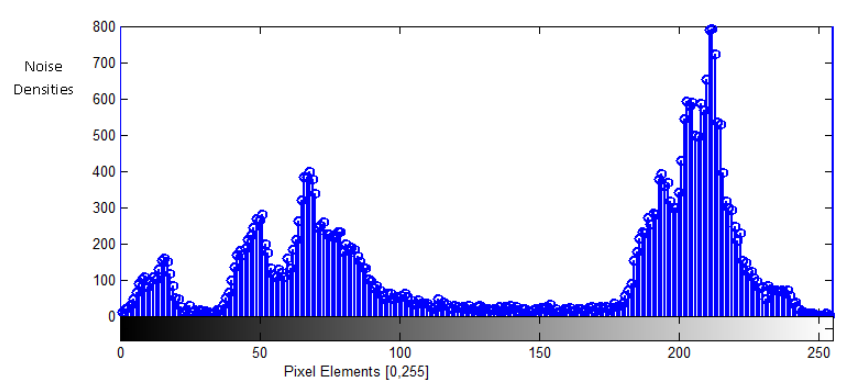

Fig. 2. Histogram of Lung CT image distorted with $50 \%$ of Salt-and-pepper noise

\begin{tabular}{r|r|r|r|r|r|r|r|r|}
\hline & 1 & 2 & 3 & 4 & 5 & 6 & \multicolumn{1}{|c|}{7} \\
\hline 1 & 255 & 255 & 164 & 166 & 168 & 166 & 255 \\
\hline 2 & 171 & 167 & 166 & 255 & 169 & 167 & 0 \\
\hline 3 & 255 & 167 & 255 & 168 & 169 & 166 & 163 \\
\hline 4 & 164 & 255 & 255 & 165 & 166 & 255 & 255 \\
\hline 5 & 0 & 255 & 255 & 163 & 0 & 162 & 255 \\
\hline 6 & 161 & 160 & 0 & 163 & 163 & 255 & 255 \\
\hline 7 & 255 & 255 & 0 & 162 & 162 & 255 & 255 \\
\hline
\end{tabular}

Fig. 3. (7x7) Noisy image pixel values

\begin{tabular}{l|l|l|l|l|l|l|l|l|l|l|}
\hline & 1 & & 2 & 3 & 4 & 5 & 6 & 7 \\
\hline 1 & & 0 & & 0 & 1 & 1 & 1 & 1 & 0 \\
\hline 2 & & 1 & 1 & 1 & 0 & 1 & 1 & 0 \\
\hline 3 & & 0 & & 1 & 0 & 1 & 1 & 1 & 1 \\
\hline 4 & 1 & & 0 & 0 & 1 & 1 & 0 & 0 \\
\hline 5 & & 0 & 0 & 0 & 1 & 0 & 1 & 0 \\
\hline 6 & 1 & 1 & 0 & 1 & 1 & 0 & 0 \\
\hline 7 & & 0 & 0 & 0 & 1 & 1 & 0 & 0 \\
\hline
\end{tabular}

Fig. 4. (7x7) Binary mask value of noisy image

$$
B(m, n)=\left\{\begin{array}{cc}
0, X(m, n)= & L_{\text {salt }} \text { or } L_{\text {pepper }} \\
1, & \text { otherwise }
\end{array}\right.
$$

where $X(m, n)$ is the pixel at location $(m, n)$ with intensity $X, B(m, n)=1$ indicates "uncorrupted pixels" to be obtained from the distorted image and $B(m, n)=0$ indicates "corrupted pixels".

\subsection{Fuzzy noise cancellation}

In this stage after creation of binary noise mask value, "corrupted pixels" marked with $\mathrm{B}(\mathrm{m}, \mathrm{n})=0$ is replaced by an estimated adjustment pixel value and then increase the window size $\mathrm{W} 2 \mathrm{~s}+1(\mathrm{~m}, \mathrm{n})$ with $(2 \mathrm{~s}+1) \mathrm{x}(2 \mathrm{~s}+1)$ of chosen noise image. Then, the number of "uncorrupted pixels" in the filtering window $\mathrm{W} 2 \mathrm{~s}+1(\mathrm{~m}, \mathrm{n})$ is calculated using

$$
G_{2 S+1}(m, n)=B(m-s: m+s, n-s: n+s)
$$

where $S$ value is choose the window size of image

After counting of uncorrupted pixel, the minimum number of "uncorrupted pixel" is less than one then expands the filtering window by one pixel [15]. This process is continued until the condition $\mathrm{G}_{2 \mathrm{~s}+1}(\mathrm{~m}, \mathrm{n})$ greater than or equal to one [18]. The uncorrupted pixels select the median pixel $\mathrm{M}(\mathrm{m}, \mathrm{n})$, given by

$$
M(m, n)=\operatorname{median}\{X(m, n)\} \text { with } B(m, n)=1
$$

The corrupted pixels select the median pixel $\mathrm{M}(\mathrm{m}, \mathrm{n})$, given by

$$
M(m, n)=\operatorname{med}\{X(m-1, n-1), X(n+1), X(n-1, m)\}
$$

After finding the value of median pixel $M(m, n)$, the local information in a $3 \times 3$ window is extracted by computing the absolute pixel difference $\alpha(m, n)$, given by

$$
\alpha(\mathrm{m}, \mathrm{n})=\operatorname{abs}(\mathrm{X}(\mathrm{m}-1: \mathrm{m}+1, \mathrm{n}-1: \mathrm{n}+1)-\mathrm{X}(\mathrm{m}, \mathrm{n}) * \text { ones }(3,3))
$$

Then the local information is described by the maximum absolute pixel difference in the $3 \times 3$ filtering window

$$
d(m, n)=\max (\alpha(m, n))
$$

In filtering stage, the Fuzzy reasoning is applied to the 


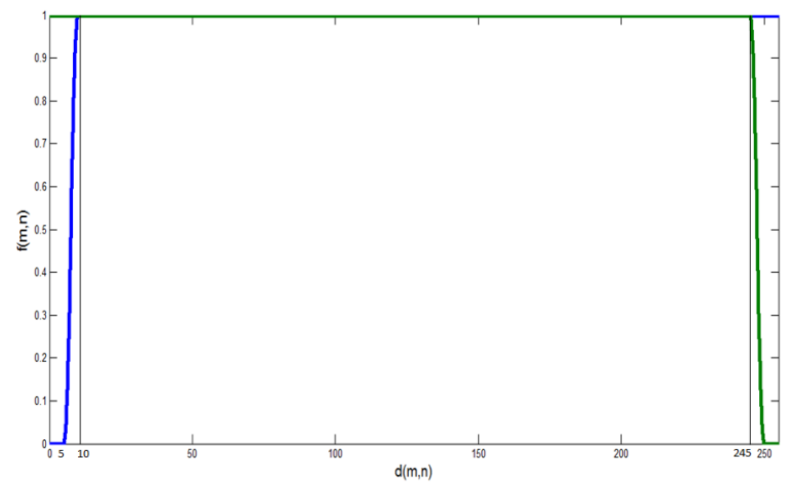

Fig. 5. Two-sided $\pi$-membership function

extracted local information $d(m, n)$. The adopted fuzzy set, which is defined by the Two-sided $\pi$-membership function(MF) $\mathrm{f}(\mathrm{m}, \mathrm{n})$ with four predefined thresholds T1(5), T2(10), T3(245) and T4(250) as shown in Fig. 5 [19].

The two-sided $\pi$-membership function [19], given by

$$
\mathrm{f}(\mathrm{m}, \mathrm{n})=\left\{\begin{array}{cc}
0, & \text { ford }(m, n) \leq T 1 \\
S(d(m, n), T 1, T 2), & \text { for } T 1<d(m, n)<T 2 \\
1, & \text { for } T 2 \leq d(m, n) \leq T 3 \\
Z(d(m, n), T 3, T 4), & \text { for } T 3<d(m, n)<T 4 \\
0, & T 4 \leq d(m, n)
\end{array}\right.
$$

Where $\mathrm{S}(\mathrm{d}(\mathrm{m}, \mathrm{n}), \mathrm{T} 1, \mathrm{~T} 2)$ is S-shaped open-right MF as shown in Fig.6, given by

$$
\begin{aligned}
& S(d(m, n), T 1, T 2) \\
& =\left\{\begin{array}{cc}
0, & \operatorname{ford}(m, n)<T 1 \\
2\left(\frac{d(m, n)-T 1}{T 2-T 1}\right)^{2}, & \text { for } T 1<d(m, n) \leq\left(\frac{T 1+T 2}{2}\right) \\
1-2\left(\frac{T 2-d(m, n)}{T 2-T 1}\right)^{2}, & \text { for }\left(\frac{T 1+T 2}{2}\right)<d(m, n) \leq T 2 \\
1, & \text { for } T 2<d(m, n)
\end{array}\right.
\end{aligned}
$$

and $\mathrm{Z}(\mathrm{d}(\mathrm{m}, \mathrm{n}), \mathrm{T} 3, \mathrm{~T} 4)$ is $\mathrm{Z}$-shaped open-left MF as shown in Fig. 7, given by

$$
Z(d(m, n), T 3, T 4)=1-S(d(m, n), T 1, T 2)
$$

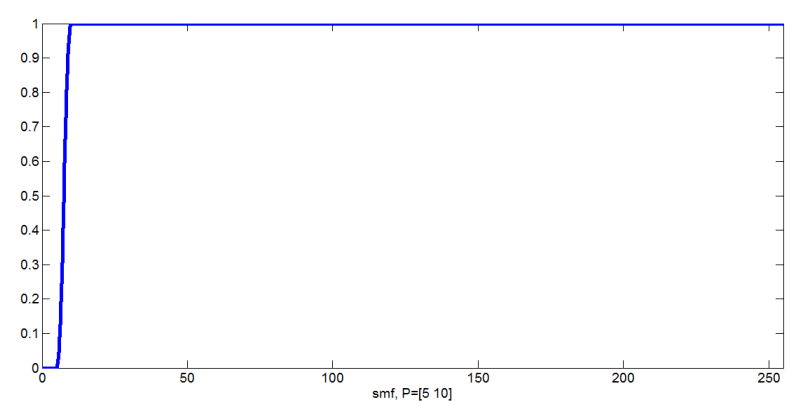

Fig. 6. S-Shaped membership function

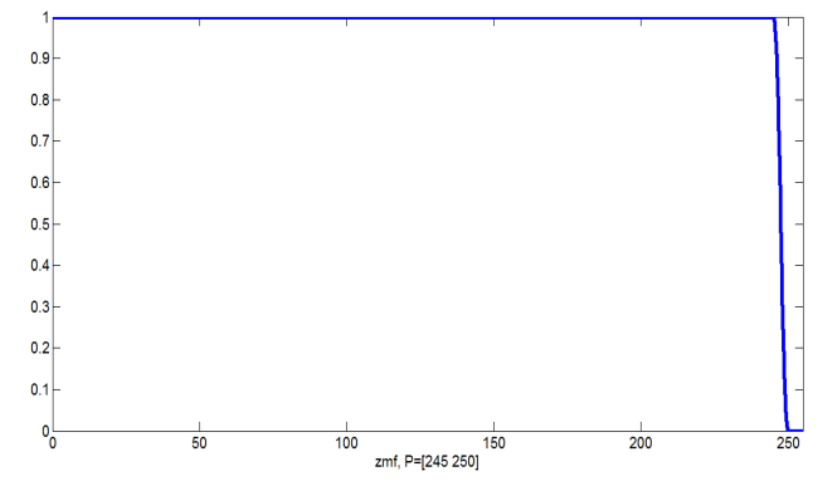

Fig. 7. Z-Shaped membership function

Where the local information $d(m, n)$ is used as the fuzzy variable and the four thresholds T1, T2, T3 and T4 are set to $5,10,245$ and 250 respectively for best performance [11]. The restored image $\mathrm{Y}(\mathrm{m}, \mathrm{n})$ is acquired by linear combination of weighted processing pixel $\mathrm{X}(\mathrm{m}, \mathrm{n})$ and median pixel $\mathrm{M}(\mathrm{m}, \mathrm{n})$ of noisy image [15].

$$
Y(m, n)=[1-f(m, n)]^{*} X(m, n)+f(m, n) * M(m, n)
$$

\section{Simulation Results and Discussion}

In this section, the performance of the proposed fuzzy based median filter was tested with medical images with noise levels are varied from $10 \%$ to $90 \%$ and compared with other existing salt-and-pepper noise removal filters. The restoration performances are quantitatively measured by several different kinds of metric value such as peak signal to noise ratio (PSNR), mean square error (MSE), RMSE, image enhancement factor (IMF), mean absolute error (MAE) and elapsed time [18].

The PSNR is most commonly used as a quality measurement for lossy compressed images. The PSNR is the ratio of the maximal power of original image and the noise power of distorted image. It is represented in the logarithmic domain because the powers of signals are usually in a wide dynamic range [6]. Its formula is given by

$$
P S N R=10 * \log _{10}\left(\frac{255^{2}}{M S E}\right)
$$

MSE is the most widely used image quality assessment based on error sensitivity [10]. It is computed by taking the average of squared intensity differences in every pixel of a reference image and a distorted image:

$$
\begin{aligned}
& M S E= \frac{\sum_{m n}(I(m, n)-Y(m, n))^{2}}{M N} \\
& I E F=\frac{\sum_{m n}(X(m, n)-I(m, n))^{2}}{\sum_{m n}(Y(m, n)-I(m, n))^{2}}
\end{aligned}
$$




$$
M A E=\frac{\sum_{m n}|(I(m, n)-Y(m, n))|}{M N}
$$

where $\mathrm{I}(\mathrm{m}, \mathrm{n})$ is Original image, $\mathrm{Y}(\mathrm{m}, \mathrm{n})$ is Denoised image $\mathrm{X}(\mathrm{m}, \mathrm{n})$-Noise image and $(\mathrm{M} \times \mathrm{N})$-size of the image.

To obtain more correct assessments, the structural simailarity (SSIM) metric which represents perceptual image quality based on the structural information [13]. SSIM is an objective image quality metric and is superior to traditional quantitative measures such as MSE and PSNR [20].

A general form of SSIM is

$$
\operatorname{SSIM}(m, n)=[l(m, n)]^{\alpha}[c(m, n)]^{\beta}[s(m, n)]^{\gamma}
$$

Where $\alpha>0, \beta>0$ and $\gamma>0$ are parameters used to adjust the relative importance of the three components and $m, n$ are image patches.

Where $1(\mathrm{~m}, \mathrm{n})$ is luminance comparison

$$
l(m, n)=\frac{2 \mu_{m} \mu_{n}+C_{1}}{\mu_{m}^{2}+\mu_{n}^{2}+C_{1}},
$$

$\mathrm{c}(\mathrm{m}, \mathrm{n})$ is contrast comparison

$$
c(m, n)=\frac{2 \sigma_{m} \sigma_{n}+C_{2}}{\sigma_{m}^{2}+\sigma_{n}^{2}+C_{2}},
$$

and $\mathrm{s}(\mathrm{m}, \mathrm{n})$ is structural comparison $s(m, n)=\frac{\sigma_{m n}+C_{3}}{\sigma_{m} \sigma_{n}+C_{3}}$

Table 1. MSE of Lung CT scan image for various filters at different noise densities

\begin{tabular}{c|c|c|c|c|c|c}
\hline $\begin{array}{c}\text { Noise density } \\
\text { in } \%\end{array}$ & SMF & AMF & DBA & PSMF & UTDBMF & PA \\
\hline 10 & 93 & 44 & 21 & 56 & 27 & 17 \\
\hline 20 & 165 & 106 & 46 & 93 & 49 & 34 \\
\hline 30 & 407 & 322 & 70 & 169 & 77 & 53 \\
\hline 40 & 1030 & 1001 & 103 & 301 & 110 & 68 \\
\hline 50 & 2422 & 2373 & 162 & 730 & 166 & 99 \\
\hline 60 & 4197 & 4522 & 253 & 1584 & 258 & 135 \\
\hline 70 & 7618 & 7665 & 445 & 7925 & 514 & 175 \\
\hline 80 & 11686 & 11688 & 768 & 11821 & 1289 & 258 \\
\hline 90 & 16839 & 16574 & 1616 & 16645 & 3126 & 540 \\
\hline
\end{tabular}

Table 2.RMSE of Lung CT scan image for various filters at different noise densities

\begin{tabular}{c|c|c|c|c|c|c}
\hline $\begin{array}{c}\text { Noise } \\
\text { density in \% }\end{array}$ & SMF & AMF & DBA & PSMF & UTDBMF & PA \\
\hline 10 & 9.67 & 6.65 & 4.62 & 7.51 & 5.28 & 4.22 \\
\hline 20 & 12.85 & 10.32 & 6.83 & 9.69 & 7.02 & 5.86 \\
\hline 30 & 20.19 & 17.94 & 8.41 & 13.00 & 8.82 & 7.32 \\
\hline 40 & 32.10 & 31.65 & 10.16 & 17.36 & 10.73 & 8.30 \\
\hline 50 & 49.21 & 48.72 & 12.74 & 27.03 & 12.90 & 9.95 \\
\hline 60 & 64.79 & 67.24 & 15.93 & 39.80 & 16.06 & 11.62 \\
\hline 70 & 87.28 & 87.55 & 21.10 & 89.02 & 22.68 & 13.26 \\
\hline 80 & 108.10 & 108.11 & 27.71 & 108.72 & 35.91 & 16.08 \\
\hline 90 & 129.76 & 128.74 & 40.19 & 129.01 & 55.91 & 23.25 \\
\hline
\end{tabular}

Where $\mathrm{C}_{1}, \mathrm{C} 2, \mathrm{C} 3$ are constants and $\mu_{\mathrm{m}}, \mu_{\mathrm{n},} \sigma_{\mathrm{m}}, \sigma_{\mathrm{n}}, \sigma_{\mathrm{mn}}$ are local sample means and standard deviations of $m$ and $n$.

The MSE, RMSE, MAE, PSNR, IEF, elapsed time in seconds and SSIM value are calculated for the proposed algorithm by varying the noise density from $10 \%$ to $90 \%$ and comparisons of performance values of various existing median based filters for lung CT scan image are tabulated in Tables 1-7.

From the Table 1, shows that the MSE value of proposed algorithm is very low compared to other existing noise removal methods even noisy densities varied from $10 \%$ to $90 \%$. The RMSE value in Table 2 shows that at high noise levels, RMSE values of existing noise removal filters are very high as compared to the proposed method. From Table 3, we illustrate that the MAE value of proposed method is very low compared to other existing noise removal method.

Table 3. MAE of Lung CT scan image for various filters at different noise densities

\begin{tabular}{c|c|c|c|c|c|c}
\hline $\begin{array}{c}\text { Noise } \\
\text { density in \% }\end{array}$ & SMF & AMF & DBA & PSMF & UTDBMF & PA \\
\hline 10 & 5.03 & 1.96 & 0.82 & 1.34 & 0.79 & 4.22 \\
\hline 20 & 5.90 & 2.60 & 1.64 & 2.31 & 1.51 & 1.47 \\
\hline 30 & 7.77 & 4.32 & 2.46 & 3.64 & 2.31 & 2.25 \\
\hline 40 & 11.64 & 8.68 & 3.37 & 5.61 & 3.20 & 2.97 \\
\hline 50 & 19.65 & 17.05 & 4.56 & 10.06 & 4.28 & 3.88 \\
\hline 60 & 30.12 & 29.74 & 6.15 & 17.41 & 5.77 & 4.84 \\
\hline 70 & 48.96 & 47.75 & 8.51 & 51.72 & 8.83 & 5.87 \\
\hline 80 & 71.22 & 70.78 & 11.66 & 73.82 & 16.70 & 7.37 \\
\hline 90 & 99.35 & 98.01 & 19.21 & 99.76 & 34.29 & 10.40 \\
\hline
\end{tabular}

Table 4. PSNR of Lung CT scan image for various filters at different noise densities

\begin{tabular}{c|c|c|c|c|c|c}
\hline $\begin{array}{c}\text { Noise } \\
\text { density in } \%\end{array}$ & SMF & AMF & DBA & PSMF & UTDBMF & PA \\
\hline 10 & 28.41 & 31.67 & 34.82 & 30.60 & 33.66 & 35.60 \\
\hline 20 & 25.95 & 27.85 & 31.43 & 28.40 & 31.19 & 32.76 \\
\hline 30 & 22.02 & 23.05 & 29.63 & 25.84 & 29.21 & 30.83 \\
\hline 40 & 18.10 & 18.12 & 27.99 & 23.33 & 27.68 & 29.74 \\
\hline 50 & 14.28 & 14.37 & 26.02 & 19.49 & 25.91 & 28.17 \\
\hline 60 & 11.91 & 11.57 & 24.08 & 16.13 & 24.01 & 26.82 \\
\hline 70 & 9.31 & 9.28 & 21.64 & 9.14 & 21.01 & 25.67 \\
\hline 80 & 7.45 & 7.45 & 19.27 & 7.40 & 17.02 & 24.02 \\
\hline 90 & 5.86 & 5.93 & 16.04 & 5.91 & 13.18 & 20.80 \\
\hline
\end{tabular}

Table 5.IEF of Lung CT scan image for various filters at different noise densities

\begin{tabular}{c|c|c|c|c|c|c}
\hline $\begin{array}{c}\text { Noise density } \\
\text { in } \%\end{array}$ & SMF & AMF & DBA & PSMF & UTDBMF & PA \\
\hline 10 & 22.96 & 50.02 & 101.98 & 38.89 & 77.30 & 124.22 \\
\hline 20 & 26.62 & 41.23 & 94.81 & 46.77 & 89.78 & 127.58 \\
\hline 30 & 16.03 & 19.89 & 92.56 & 39.27 & 84.49 & 123.69 \\
\hline 40 & 8.46 & 8.81 & 84.75 & 29.44 & 80.37 & 126.50 \\
\hline 50 & 4.54 & 4.63 & 67.35 & 14.88 & 66.39 & 110.69 \\
\hline 60 & 3.09 & 2.92 & 51.58 & 8.22 & 50.95 & 98.70 \\
\hline 70 & 2.01 & 2.23 & 34.57 & 1.94 & 29.65 & 87.06 \\
\hline 80 & 1.50 & 1.49 & 22.86 & 1.48 & 13.62 & 67.91 \\
\hline 90 & 0.01 & 1.18 & 12.22 & 1.18 & 6.29 & 36.55 \\
\hline
\end{tabular}


Table 6. Elapsed time of Lung CT scan image for various filters at different noise densities

\begin{tabular}{c|c|c|c|c|c|c}
\hline $\begin{array}{c}\text { Noise } \\
\text { density in } \%\end{array}$ & SMF & AMF & DBA & PSMF & $\begin{array}{c}\text { UTD } \\
\text { MF }\end{array}$ & PA \\
\hline 10 & 1.85 & 2.27 & 7.34 & 2.12 & 2.38 & 5.16 \\
\hline 20 & 1.77 & 2.58 & 7.62 & 2.95 & 2.52 & 5.94 \\
\hline 30 & 2.06 & 2.62 & 5.94 & 2.89 & 2.48 & 6.71 \\
\hline 40 & 1.95 & 1.74 & 7.02 & 2.47 & 2.62 & 8.17 \\
\hline 50 & 1.76 & 2.45 & 6.96 & 2.58 & 2.41 & 8.15 \\
\hline 60 & 1.73 & 1.76 & 6.98 & 2.89 & 2.52 & 9.34 \\
\hline 70 & 1.78 & 2.71 & 7.69 & 2.15 & 3.46 & 11.52 \\
\hline 80 & 1.84 & 1.77 & 6.21 & 2.51 & 3.49 & 13.65 \\
\hline 90 & 1.76 & 2.04 & 5.98 & 2.61 & 3.34 & 14.40 \\
\hline \multicolumn{7}{|r|}{}
\end{tabular}

Table 7. SSIM of Lung CT scan image for various filters at different noise densities

\begin{tabular}{c|c|c|c|c|c|c}
\hline $\begin{array}{c}\text { Noise } \\
\text { density in \% }\end{array}$ & SMF & AMF & DBA & PSMF & UTDBMF & PA \\
\hline 10 & 0.7932 & 0.9061 & 0.9763 & 0.9583 & 0.9776 & 0.9840 \\
\hline 20 & 0.7478 & 0.8732 & 0.9460 & 0.9171 & 0.9540 & 0.9574 \\
\hline 30 & 0.6276 & 0.7470 & 0.9141 & 0.8493 & 0.9258 & 0.9375 \\
\hline 40 & 0.4427 & 0.5187 & 0.8772 & 0.7114 & 0.8915 & 0.8987 \\
\hline 50 & 0.2460 & 0.2868 & 0.8305 & 0.4957 & 0.8362 & 0.8539 \\
\hline 60 & 0.1362 & 0.1444 & 0.7640 & 0.2987 & 0.7395 & 0.8114 \\
\hline 70 & 0.0667 & 0.0713 & 0.6823 & 0.0677 & 0.5420 & 0.7664 \\
\hline 80 & 0.0352 & 0.0362 & 0.5955 & 0.0366 & 0.3021 & 0.7004 \\
\hline 90 & 0.0143 & 0.0149 & 0.4473 & 0.0161 & 0.1378 & 0.5746 \\
\hline
\end{tabular}

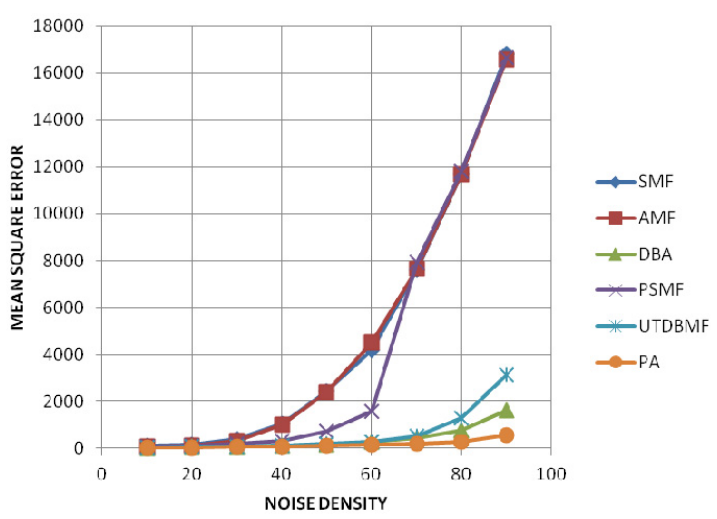

Fig. 8. Comparison of MSE value of different algorithms for Lung CT scan image

The PSNR, IEF and SSIM values of proposed method in Table 4, Table 5 and Table 7 shows that at higher noise levels the PSNR, IEF and SSIM values of existing methods are very low as compared to the proposed method. From all the performance values of proposed method, it is clearly observed that the performance of the proposed algorithm is moderately significant than the existing algorithms at both low and high noise densities. The performance analysis against noise densities for lung CT scan image is also done with help of plot shown in Figs. 8-13.

The difference in detail preservation performance of the proposed method may be better observed by looking at the output images of the different methods [19]. For this purpose, the Lung CT scan image shown in Fig. 14(a) of

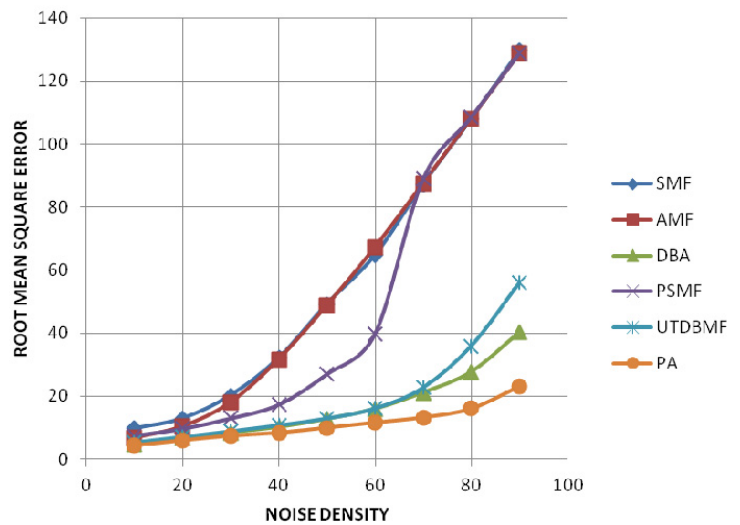

Fig. 9. Comparison of RMSE value of different algorithms for Lung CT scan image

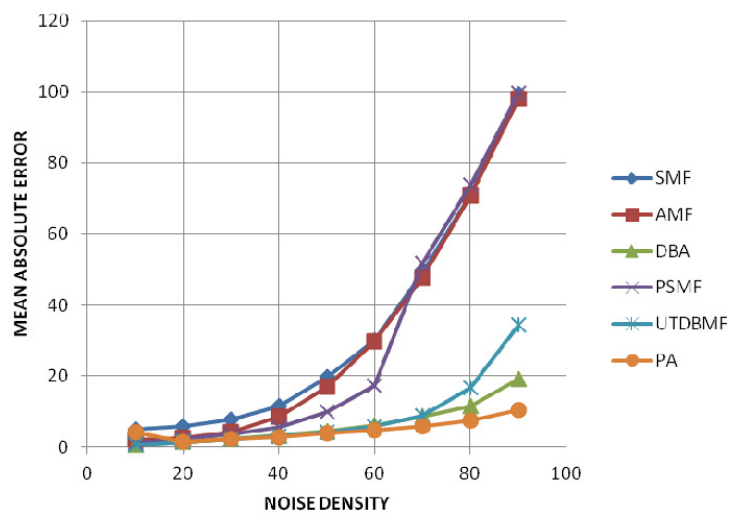

Fig. 10. Comparison of MAE value of different algorithms for Lung CT scan image

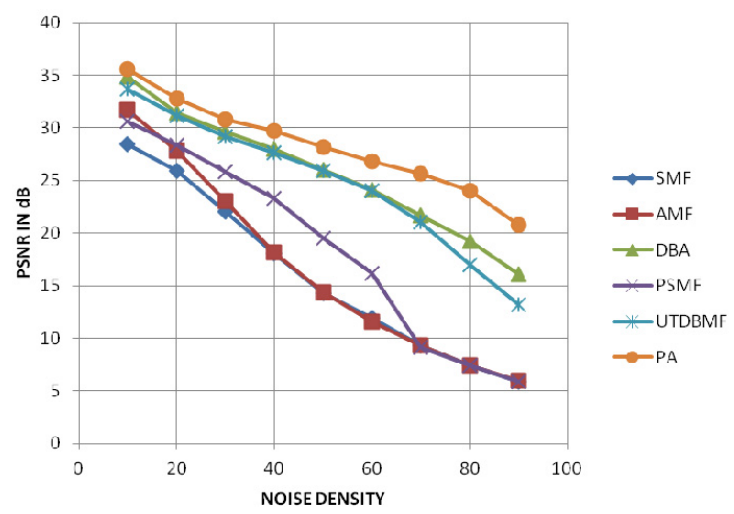

Fig. 11. Comparison of PSNR value of different algorithms for Lung CT scan image

corrupted by $80 \%$ noise and noise image shown in Fig. 14 (b) is restored by using different median based filters. Fig. 14(c)-14(h) shows the output images of the different methods. It is observed from this figure that performance of the SMF and AMF are very close to each other. Some noise mark are easily visible in the output images of these two filters. The output images of the DBA, PSMF and UTDBMF are almost indistinguishable from each other 


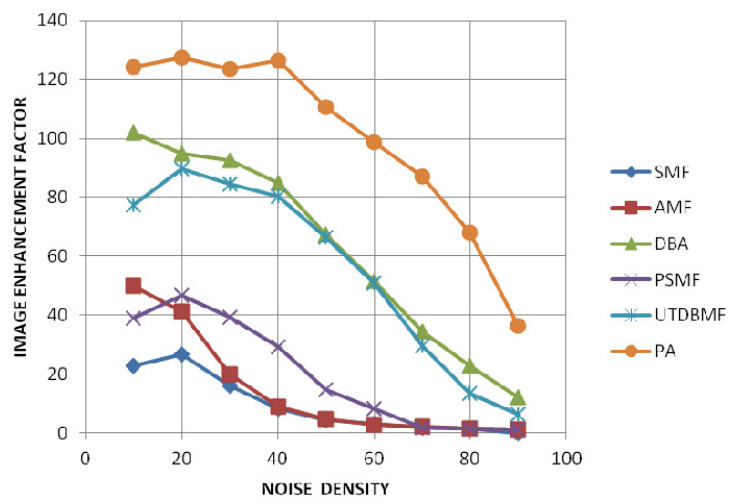

Fig. 12. Comparison of IEF value of different algorithms for Lung CT scan image

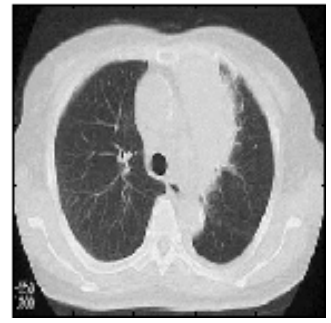

(a)

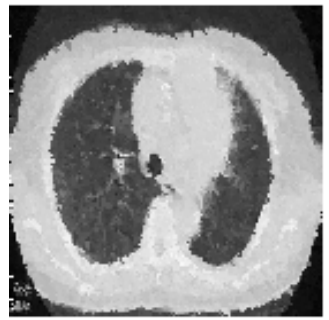

(e)

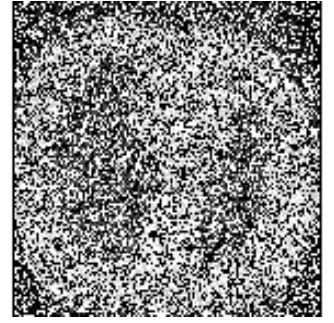

(b)

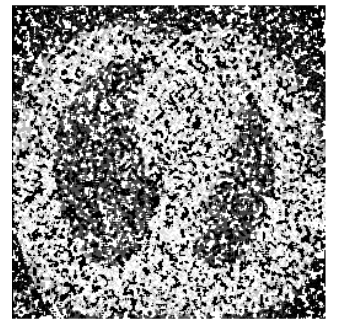

(f)

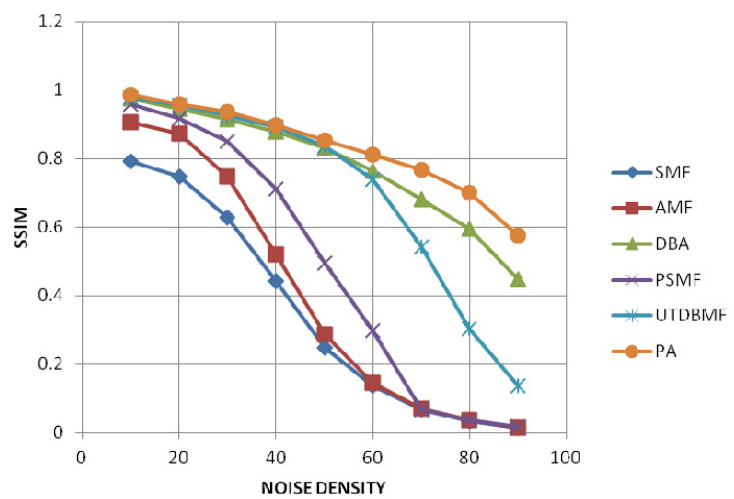

Fig. 13. Comparison of SSIM value of different algorithms for Lung CT scan image

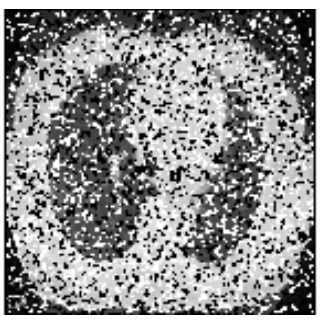

(c)

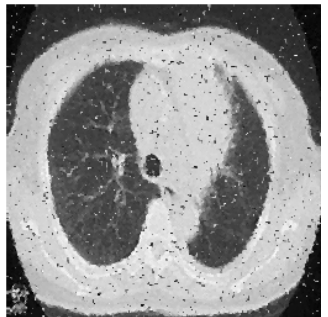

(g)

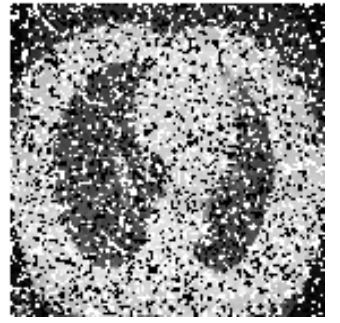

(d)

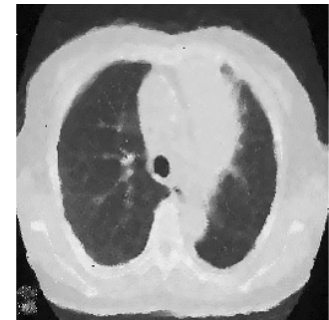

(h)

Fig. 14. (a) Original Lung CT scan image; (b) Corrupted image with 70\% of salt-and-pepper noise; (c) SMF image; (d) AMF image; (e) DBA image; (f) PSMF image; (g) UTDBMF image and (h) Proposed filtered image.

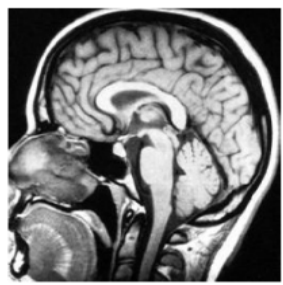

(a)

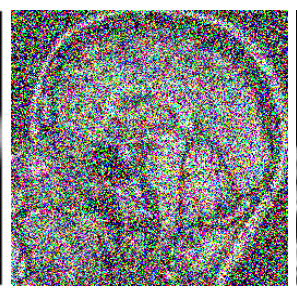

(b)

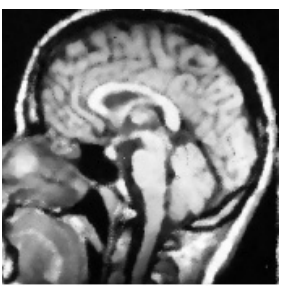

(c)

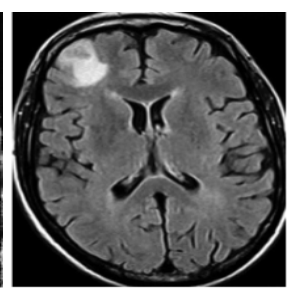

(d)

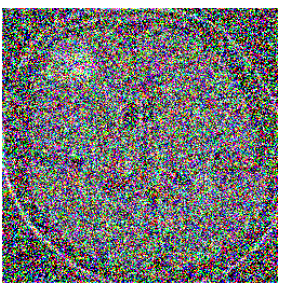

(e)

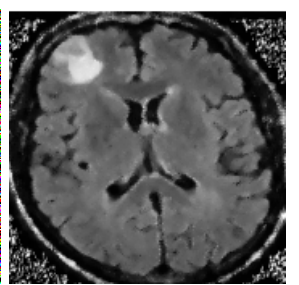

(f)

Fig.15. (a) Original MRI scan brain image; (b) 80\% noise image; (c) Proposed filtered image; (d) Original CT scan brain image; (e) $80 \%$ noise image (f) Proposed filtered image

and they are significantly better than those of the SMF and AMF. They show very good noise removal performance but considerably blur the small details of the image. It is observed that the proposed algorithm yields much better preservation performance [15].

The PSNR value of proposed filter is high compared to existing methods as shown in Fig. 10.
Fig. 15 show the reconstructed results of MRI scan brain corrupted with $80 \%$ of noise of various filters [17]. From the restored results the proposed filter produce excellent quality image for high noise density. From the Table 6 and Fig. 16, we observe that the proposed filter provides better trade off among elapsed time. 


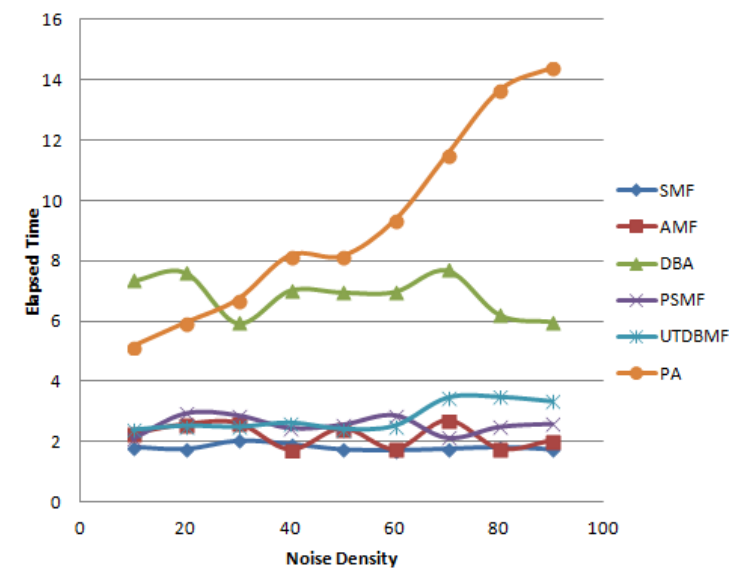

Fig. 16. Comparison of elapsed time value of different algorithms for Lung CT scan image

\section{Conclusion}

In this paper, a proposed fuzzy based median filtered algorithm has been implemented for enhancing the different medical images in terms of removal of salt-andpepper noise from highly corrupted noisy image. The extracted local information for optimal performance is achieved by two-sided $\pi$-MF compare with other fuzzy membership function. Results show that the proposed algorithm improves the metric values in comparison with other existing algorithms in terms of higher PSNR, IEF and SSIM for noise densities from $10 \%$ to $90 \%$. In addition, the moderately elapsed time and easy implementation for image denoising methods.

\section{References}

[1] Zhang and Z. wang," Impulse noise detection and removal using fuzzy Techniques," ELEECTRONICS LETTER, vol. 33, no. 5, pp. 378-379, February 1997.

[2] Esakkirajan, S., T. Veerakumar, A. N. Subramanyan and C. H. PremChand," Removal of High Density Salt and Pepper Noise Through Modified Decision Based Unsymmetric Trimmed Median Filter," IEEE Signal Processing Letters, vol. 18, no. 5, pp. 287-290, May 2011.

[3] Gonzalez, R.C. and R.E. Woods, Digital Image Processing. Printice-Hall, 2002.

[4] F. Farbiz and M. B. Menhaj, "Fuzzy Techniques in Image Processing,” New York: Springer-Verlag, 2000.

[5] Kenny Kal Vin Toh, and Nor Ashidi Mat Isa, "Noise adaptive fuzzy switching median filter for salt-and pepper noise reduction," IEEE signal processing letters, vol. 17, no. 3, pp. 281-284, March 2010.

[6] K. K. V. Toh, H. Ibrahim, and M. N. Mahyuddin, "Salt-and-pepper noise detection and reduction using fuzzy switching median filter," IEEE Trans. Con- sumer Electron., vol. 54, no. 4, pp. 1956-1961, Nov. 2008.

[7] Kenny Kal Vin Toh, and Nor Ashidi Mat Isa, "Cluster-Based Adaptive Fuzzy Switching Median Filter for Universal Impulse Noise Reduction," IEEE Transaction on consumer electronics, vol. 56, no. 4, pp. 2560-2568, November 2010.

[8] P. E. Ng and K.-K. Ma, "A switching median filter with boundary discriminative noise detection for extremely corrupted images," IEEE Trans. Image Processing, vol. 15, no. 6, pp. 1506-1516, June 2006.

[9] Srinivasan K.S and D. Ebenezer, "A new fast and efficient decision-based algorithm for removal of high-density impulse noises," IEEE Signal Process. Lett.vol.14, no.3, 189 -192, March 2007.

[10] Schulte, S V. De Witte, Nachtegael, M D. Van der Weken, and Kerre EE, "Fuzzy random impulse noise reduction method," Fuzzy Sets and Systems, vol. 158, no. 3, pp. 270-283,Feb. 2007.

[11] Smail Akkoul, Roger Ledee, Remy Leconge and Rachid Harba, "A New Adaptive Switching Median Filter," IEEE Signal Processing Letters, vol.17,no.6, pp. 587-590, April 2010.

[12] R. Pushpavalli and G. Sivaradje, "Nonlinear Filtering Technique for Preserving Edges and Fine Details on Digital Image," International Journal of Electronics and Communication Engineering and Technology, vol. 3, no. 1, pp. 29-40, January 2012.

[13] A. Fabijanska and D. Sankowski, "Noise adaptive switching median-based filter for impulse noise removal from extremely corrupted images," IET image processing, vol.5, no.5, pp.472-480, July 2010.

[14] W.K. Pratt, Digital Image Processing, Wiley, 1978.

[15] S. Zhang and M. A. Karim, "A new impulse detector for switching median filters," IEEE Signal Process. Lett., vol.9, no.11, pp. 360-363, Nov. 2002.

[16] H.-L. Eng and K.-K. Ma, "Noise adaptive soft switching median filter," IEEE Trans. Image Processing, vol.10, no.2, pp. 242-251, Feb. 2001

[17] Pei-Eng Ng and Kai - Kuang Ma, "A Switching median filter with boundary Discriminative noise detection for extremely corrupted images," IEEE Transactions on image Processing, vol.15, no.6, pp.1500-1516, June 2006.

[18] Zhou Wang and David Zhang," Progressive Switching Median Filter for the Removal of Impulse Noise from Highly Corrupted Images," IEEE Transactions on Circuits and Systems - II: Analog and Digital Signal Processing, vol. 46, no. 1, pp.78-80.January 1999.

[19] J.S.R.Jang, C. T. -Sun and E. Mizutani, Neuro-Fyzzy and Soft Computing, Printice-Hall, 1997.

[20] Z. Wang and A. C. Bovik, "Image quality assessment: From error visibility to structural similarity," IEEE Trans. Image Processing, vol. 13, no.4, pp. 600-612, Apr. 2004.

[21] M.Omair Ahmad and Duraisamy Sundararajan.”A 
Fast Algorithm for Two-Dimensional Median Filtering." IEEE Transactions on Circuits and System, vol.CAS-34, no.11, pp.1364-1374, Nov.1987.

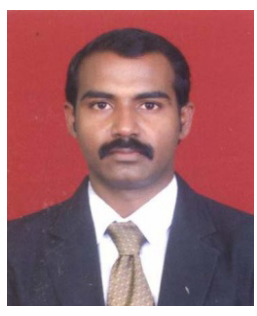

G. Elaiyaraja is currently working as an Assistant Professor in the Department of Electronics and Communication Engineering, Adhiparasakthi College of Engineering, G.B. Nagar, Vellore Tamilnadu, India since 2007.He received his bachelor degree from university of madras, Adhiyamaan of College of Engineering, Hosur in 1999 and Master degree from college of Engineering, Guindy, Anna University Chennai in 2004. He is currently doing his Ph.D under Anna University, Chennai, Tamilnadu, India. He has 14 years teaching experience. His research interest is digital image processing, nonlinear filters, and digital signal processing.

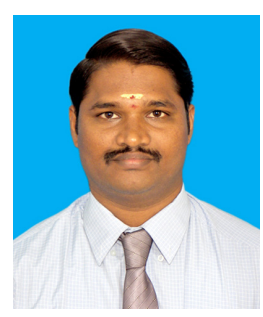

N.Kumaratharan is currently working as a Professor in the Department of Information and Technology, Sriperumbudur, Tamilnadu, India. He received his bachelor degree from University of Madras in 2001 and Master degree from college of Engineering, Guindy, in 2004. He received his Ph.D Degree in Electronics and Communication Engineering from Pondicherry University in 2010. His research interest includes wireless sensor networks, wireless and broadband communication, digital signal processing and digital image processing. He has published several papers in international journals and international Conferences. He is also member of review committees for many national and international journals. 